\title{
A Survey on Dataset Recognition of 3D Face with Missing Parts
}

\author{
Madhura Patil \\ ME Student, Department of Computer Engineering, Sinhgad Academy of Engg. Pune, Maharashtra, India
}

\begin{abstract}
The 3D face propose and try a unique answer for 2D face acknowledgment that backings precise face coordinating and give the immaculate exact result. To get the precise face representation we first concentrates key purposes of the 3-D profundity picture of the face and after that measures how the face profundity changes along facial bends associating sets of key focuses. The Face demeanor assessed by inadequate examination of facial bends characterized crosswise over in lier sets of coordinating key focuses in the middle of test and Gallery checks. In the proposed methodology, recognizing characteristics face are caught by to begin with separating key purposes of the 3D profundity picture and afterward measuring how the face profundity changes along facial bends uniting sets of key focuses. Face correlation is ascertained by looking at facial bends crosswise over in lier sets of key focuses that match between exhibition checks. Thus, facial bends of the exhibition sweeps are connected with a saliency measure all together to recognize bends that model describing attributes of some subjects from bends that are as often as possible saw in the face of a wide range of subjects. The acknowledgment of face is assessed by utilizing v2.o challenge.
\end{abstract}

Keywords: Face Recognition Grand Challenge (FRGC), Depth image, Facial Curves.

\section{Introduction}

Three-dimensional face recognization (3D face acknowledgment) is a methodology of facial acknowledgment routines in which the three-dimensional geometry of the human face is utilized. It has been demonstrated that 3D face acknowledgment techniques can accomplish altogether higher precision than their 2D partners, matching unique mark recognition.3D face acknowledgment can possibly accomplish preferred exactness over its 2D partner by measuring geometry of unbending elements on the face. This keeps away from such pitfalls of 2D face acknowledgment calculations as change in lighting, diverse outward appearances, make-up and head introduction. Another methodology is to utilize the 3D model to enhance precision of conventional picture based acknowledgment by changing the head into a known perspective. Also, most range scanners procure both a 3D lattice and the comparing surface. This permits consolidating the yield of unadulterated 3D matchers with the more customary 2D face acknowledgment calculations, consequently yielding better execution. FACE acknowledgment utilizing 3-D sweeps of the face has been as of late proposed as an option or correlative answer for routine 2-D face acknowledgment methodologies dealing with still pictures or recordings. Indeed, confront representations in view of 3-D information are relied upon to be a great deal more vigorous to posture changes and light varieties than 2-D pictures, in this way permitting exact face acknowledgment likewise in genuine applications with unconstrained securing. In such a case, test examines zone obtained in unconstrained conditions that may prompt missing parts (no frontal posture of the face, or to impediments because of hair, glasses, scarves, hand signals, and so forth. These challenges are further honed by the late coming of 4-D scanners (3-D in addition to time) fit for getting worldly groupings of 3-D filters. Truth be told, the elements of facial developments caught by these gadgets can be valuable for much application additionally builds the securing commotion and the variability in subjects' stance. In outline, notwithstanding the examination and useful significance that incomplete face coordinating arrangements are increasing, only a couple works have unequivocally tended to the issue of 3-D face acknowledgment for the situation in which a few sections of the facial outputs are absent. Numerous coordinating issue a raised genuine word application.

Numerous impediment issue happened in 3D face. To take care of this issue 3D face acknowledgment is proposed here. The fundamental mechanical confinement of 3D face acknowledgment strategies is the securing of $3 \mathrm{D}$ picture, which more often than not requires a reach camera. Then again, numerous pictures from diverse points from a typical camera may be utilized to make the 3D model with noteworthy post-preparing. This is additionally a motivation behind why $3 \mathrm{D}$ face acknowledgment techniques have risen fundamentally later than 2D systems. As of late business arrangements have executed profundity observation by anticipating a lattice onto the face and incorporating video catch of it into a high determination 3D model. This takes into account great acknowledgment exactness with ease offthe-rack parts. This procedure coordinate the all the present in the face. It coordinates the entire face. It gives the entire face representation. A novel geometric edge work for dissecting with particular objectives of looking at, coordinating, and averaging their shape .The facial surface by outspread bends radiating from the nose tips and utilize versatile shape examination of these bends. Distinctive information bases is utilized assess the exhibitions FRGCv2, GavabDB and Bosporus, every representing an alternate sort challenge.

Worldwide 3-D face representations for incomplete face coordinating have been proposed in a predetermined number of works. In standard representation of the face is proposed which misuses the isometric invariance of the face surface to oversee missing information got by arbitrarily expelling

\section{Volume 4 Issue 12, December 2015}




\section{International Journal of Science and Research (IJSR) \\ ISSN (Online): 2319-7064}

Index Copernicus Value (2013): 6.14 | Impact Factor (2014): 5.611

zones from frontal face checks. On a little database of 30 subjects they reported high. One of the approaches to do this is by contrasting chose facial components from the picture and a facial database. It is regularly utilized as a part of security frameworks and can be contrasted with different biometrics, for example, unique mark or eye iris Recognization frameworks.

\section{Related Work}

Existing framework highlight extraction is done utilizing entire face representation. In 3D face representation utilizing Blends of arrangements in these two classifications are too conceivable and additionally multimodal approaches that join together 2-D and 3-D methods. Review 3-D face acknowledgment arrangements that have been proposed and assessed utilizing facial sweeps with missing part. It doesn't fulfill the nature of entire face representation. Worldwide 3$\mathrm{D}$ face representations for incomplete face coordinating have been proposed in a set number of works. The characteristics of entire face not fulfilled in the current framework. The profundity of picture not caught in the current framework. Handling the issue from an inverse point of view, a few techniques partition the face into areas and attempt to confine the match to uncorrupted parts of the face. Couple of facial points of interest can be precisely identified in a programmed path-from three to ten are at manual help. On account of incomplete face sweeps, up to half of these focuses are ordinarily no noticeable, so that depiction of such focuses and of their connections is of constrained viability for face acknowledgment. The principle mechanical constraint of 3D face acknowledgment techniques is the procurement of $3 \mathrm{D}$ picture, which for the most part requires a range camera. Numerous impediment issue in the 3D face representation. The correlation with database does not support the huge nature of information. The entire face does not anticipate in existing framework. The nature of the picture does not fulfill in existing methodology. In existing framework different database has been utilized. Yet, this database did not bolster all information missed.

\section{System Preliminaries}

\section{A .Capture Image}

Facial element limitation is a critical in numerous resulting errands, for example, face acknowledgment, posture standardization ,appearance comprehension and face following.

\section{B. Preprocessing}

To decrease the computational weight, Down example the high determination face pictures

\section{Smoothing}

This method is utilized to dispose of the light that are happened in the picture normally by regular mutilation $\mathrm{s}$. Smoothing uproots transient varieties, or "clamor" to uncover the critical hidden unadulterated type of the information.

\section{Normalization}

Standardization is a procedure that progressions the scope of pixel power values. Applications incorporate photos with poor contrast because of glare, for instance. Standardization is at times called difference extending. In more broad fields of information handling, for example, computerized sign preparing, it is alluded to as element range extension. The motivation behind element range extension in the different applications is typically to bring the picture, on the other hand other sort of sign, into a reach that is more well known or typical to the faculties, thus the term standardization.

\section{E. Key points Repeatability}

A face coordinating methodology that joins spatial imperatives for key points coordinating with a unique detailing of the saliency of facial bends for display sweeps, in this way permitting weighted match of diverse facial bends. Key points extricated from diverse facial sweeps of the same individual are relied upon to be found roughly in the same positions on the face.

\section{G. Facial Curves}

While the larger part of face acknowledgment specialists will concur that execution can be essentially expanded, there is an argumentative level headed discussion about how to accomplish this objective. This investigation recommends that, contingent upon the particular application, an exchange off in the middle of exactness and computational time can be found. Comparative results were acquired for the UND dataset. On this dataset, our outcomes are contrasted and those reported in and that utilized a test setup like that proposed in this work. Table $\mathrm{V}$ abridges the assessment utilizing rank-1 RR as execution pointer. Results plainly show that our methodology is equipped for accomplishing or enhancing the cutting edge execution for every one of the classes of sweeps but one (i.e., looking-down). As a general conduct of the methodologies under examination, an entirely vast distinction in perceiving left and right side sweeps can be noted for this dataset (around 11\%, 14\% and 16\% reduction, separately, for our work and the methodologies and Measuring the yaw revolution for the left and right side sweeps, can acquired a normal point of around 50 and 70 , separately. These turn points are lower than the ostensible qualities reported in the database depiction, and the distinction of around 20 between the yaw turns of left and right sweeps can be inspiration for the distinctive exactness appeared in the acknowledgment.

\section{Key Points of Depth Facial Images}

Recognition of significant key points from profundity pictures of the face is the initial step of the proposed approach. Before the location of key points, the 3-D outputs experience to an inflexible 3-D change (revolution and interpretation) in order to standardize their posture to a typical reference outline. This is a nontrivial issue independent from anyone else; furthermore, a few methodologies have been as of late reported for the particular area of 3-D faces [3], [4]. In this manner, we depend on existing strategies to infer a couple of historic points of the face that are perceptible with a decent 


\section{International Journal of Science and Research (IJSR) \\ ISSN (Online): 2319-7064}

Index Copernicus Value (2013): 6.14 | Impact Factor (2014): 5.611

exactness likewise in acquisitions with extensive posture varieties (up to around 70/80 degrees), and use them to perform face arrangement. This is proficient by registering the 3-D revolution and interpretation change that minimizes the mean Euclidean separation of relating points of interest. The utilized historic points are, to be specific, the nose tip, the inward and external eye corners, what's more, the mouth corners. To recognize the nose tip we utilized the methodology in [8], that first characterizes a vitality measure to portray the nearby conveyance of neighboring focuses and recognize the applicants nose tips, then concentrates two feeble neighborhood includes and chooses from the hopefuls the genuine nose tip utilizing a various leveled sifting plan. The methodology takes a shot at focuses mists and is powerful to revolution, interpretation, gaps and anomalies. Keeping in mind the end goal to uproot focuses that don't have a place with the face, the nose tip is moreover utilized as focal point of a circle of focuses that fall inside of a separation of $100 \mathrm{~mm}$, forgetting every one of the focuses surpassing this range (an outright separation is utilized as a part of that real measures of the face size are essential to separate between diverse subjects [9]). The remaining historic points are inferred utilizing a variation of the arrangement proposed in [10], [11], where the shape record and the corne rness are both removed from 3-D information and used to portray hopefuls areas. Extents of the face are then used to choose the areas that compare to the looked milestones. These procedures can be effectively executed and empower the extraction of facial points of interest with low computational expense (i.e., not exactly 2 seconds are required to separate the points of interest on a Centrino Twosome 2.2 GHZ CPU, with 2 GB memory).

Once the points of interest are identified and utilized for instating the arrangement handle, the stance of the 3-D countenances is then refined concurring to an unbending change utilizing the ICP calculation [7].Thusly, 3-D focuses are enlisted as for a reference model got by averaging a chose set of FRGC v1.0 examines with unbiased expression and frontal stance. Subsequently, the obtained outputs are standardized to a typical stance and profundity pictures of the face are separated. Some further preprocessing steps are connected to the profundity pictures before investigating them for key points location. These record for spikes evacuation utilizing middle sifting in the - direction, filling of little openings with cubic interjection, what's more, 3-D sweeps resampling on a uniform square framework at 0.7 mm determination.

The subsequent profundity pictures are utilized to concentrate face key points by running the SIFT keypoints indicator [12]. At the point when connected to profundity pictures, SIFT keypoints distinguish blobs of profundity qualities, relating to scale-space extrema of the scale standardized contrast of Gaussians administrator. Since blobs top to bottom sweeps relate to districts whose profundity qualities contrast contrasted with the encompassing recognized key points compare to neighborhood bulges/interruptions of the face surface. Contingent upon the size of discovery, the key points may relate to significant or minor bulges: the tip of the nose, the button, the cheeks are commonly identified at a coarse scale, while at better scales key points are limited in correspondence to overlap, wrinkles, edges and scores of the face. These involve the expression lines, for example, the naso-labial and bucco-labial creases, the brow and eye wrinkles, the tear troughs, the external and inward canthi [13]. For each key point, the relating SIFT descriptor is additionally processed utilizing a 16 pixel support. This little bolster size, gives our methodology the obliged heartiness to missing parts, coming about, in the meantime, adequate to set up predictable correspondences between key point descriptors. The properties of the SIFT descriptor make it equipped for giving a reduced yet intense nearby representation of the profundity picture what's more, as an outcome, of the face surface. The 3-D face sweeps are orchestrated into five sections, every segment indicating three outputs of the same subject: the display examine, one frontal unbiased test filter and one side test filter. In every one of the cases, the key points recognized.

\section{A. Key points Clustering}

As indicated by the SIFT model, key points are separated at distinctive scales, the scale parameter relating to the standard deviation of the smoothing Gaussian part. Recognized key points disseminate unpredictably over the face surface: in some parts of the face they are more thickly disseminated than in other parts. Totals of close key points generally happen in correspondence to locales of the face surface described by high spatial recurrence segments regularly because of facial wrinkles in the mouth, eyes and nose locales. Keeping in mind the end goal to hold just the most steady, dreary and educational key points, the arrangement of distinguished key points is liable to bunching, as indicated by the various leveled bunching model [1].

Specifically, this is performed on the arrangement of all key points, paying little mind to the scale at which they are distinguished. Various leveled bunching gatherings information over an assortment of granularity levels by making a group tree where bunches at one bland level are joined to shape groups at the following level. This permits us to decide the most proper level of key points grouping in three stages:

- First, the spatial separation (Euclidean separation in the 2D plane of the profundity picture) between each pair of key points is processed;

- Then, the key points are iteratively gathered into a twofold, progressive bunch tree. At every cycle step, the pair of nearest key points (or bunches) are assembled together. In this stride, a solitary linkage procedure [1] is received for the calculation of the separation between groups (that is, the most brief Euclidean separation between components in two bunches is expected as group separation);

- Finally, the choice of where to cut the progressive tree into bunches is taken. In this stride, branches off the base of the progressive tree are pruned, and all the key points underneath every slice are doled out to a solitary bunch. This makes a parcel of the information. The groups are made by recognizing common groupings in the various leveled tree and ceasing the total procedure when the spatial sweep of the bunch drops beneath an edge of spatial rationality (set equivalent to $10 \mathrm{~mm}$ in our examinations).

\section{Volume 4 Issue 12, December 2015}




\section{International Journal of Science and Research (IJSR) \\ ISSN (Online): 2319-7064}

Index Copernicus Value (2013): 6.14 | Impact Factor (2014): 5.611

When the bunches are distinguished, the most stable key point of every bunch (i.e., the key point identified at the biggest scale) is held and used to speak to the locale of the face around the bunch focus. Regardless, the quantity of identified bunches has been set to be no lower than 20, in order to allow an adequate number of key points to be utilized for the examination of two facial checks.

\section{B. Key points Repeatability}

The thought to ground the face representation on an inadequate and versatile arrangement of consequently identified key points depends on the suspicion of intra subject key points repeatability: Key points separated from diverse facial outputs of the same individual are anticipated that would be found around in the same positions on the face. Since the restriction of key points separated by handling a face check through the SIFT finder just relies on upon the geometry of the face surface, these key points are not ensured to relate to particular significant points of interest of the face. For the same reason, the location of key points on two face examines of the same individual ought to respect the distinguishing proof of the same purposes of the face, unless the state of the face is adjusted by real impediments or non neutral outward appearances. To test the repeatability of key points location, we took after the methodology proposed in [25] utilizing the 3-D checks as a part of the Spring2003 envelope of the FRGC v2.0 dataset [5] (i.e., 943 threedimensional sweeps with nonpartisan articulation of 275 people altogether). This dataset is additionally alluded to as the FRGC v1.0 dataset, and is proposed to be utilized as preparing information for techniques confirmed on the FRGC v2.0 test set. A measure of the correspondence of the area of key points identified in two face outputs is gotten by ICP enlistment [34]. Appropriately, the 3-D confronts fitting in with the same individual are naturally enlisted and the blunders between the closest neighbors of their key points (one from every face) are recorded. Fig. 4 demonstrates the after effects of our key point repeatability test, by reporting the combined rate of repeatability as an element of expanding estimations of the separation. The repeatability comes to $57 \%$ for appearances with nonpartisan expression at a mistake of $5 \mathrm{~mm}$ (with a normal number of 62 key points recognized per check). This estimation of repeatability is lower than the worth reported in [6] for their 3-D key points indicator. This is essentially propelled by the much bigger number of key points recognized in [6] (around 400 key points per face): the higher the quantity of key points identified on two sweeps of the same subject, the lower the separation between a non specific key point on the first sweep and its nearest key point on the second check. On the other hand, it ought to be seen that, in our methodology, even a little number of key points repeatable with a little spatial blunder is adequate to determine a spellbinding representation of the face in light of facial bends (see the following Section for subtle elements on facial bends extraction). This instinct has been affirmed in the tests, where compelling face acknowledgment has been gotten with various coordinating key points running from as few as 8 to more than.

\section{Face Matching}

Given two face checks, the choice about whether they speak to the same individual or not depends on the examination of the facial bends identified on the two outputs. Then again, keeping in mind the end goal to bolster exact acknowledgment, correlation of facial bends ought to too consider some geometric imperatives about the position of facial bends on the face and additionally some idea of bend saliency to represent the way that facial bends are definitely not just as discriminative for face acknowledgment.

\section{A. Spatial Consistency and Key points Matching}

Given two face models, their examination is performed by mutually coordinating the key points and the facial bends under the limitation of predictable spatial course of action of relating key points on the two face models. To begin with, SIFT descriptors of the key points recognized in the test and the exhibition are looked at, so that for each key point in the test, a hopeful relating key point in the display is recognized. Specifically, a key point in the test is alloted to a key point in the display, on the off chance that they match one another among all key points, that is if it is closer to than to some other key point in the display and is closer to than to some other key point in the test. For this reason, nearness of key points is measured through the Euclidean separation between 128-dimensional SIFT descriptors connected with the key points.

\section{B. Saliency of Facial Curves}

Calculation of the likeness between a test and a display examine by averaging separations of comparing facial bends measured through (2) would bring about poor acknowledgment precision. Inspirations are twofold and identify with the unpredictability and saliency of facial bends. Facial bends identified on a face filter may have altogether different shapes. As Fig. 6 demonstrates, some facial bends (e.g., the blue bend spoke to in the lower right plot) can estimated well a smooth straight line, though other bends (e.g., the green bend in the lower left plot) show more mind boggling examples. Accordingly, the measure of the separation between two bends processed by is not straightforwardly illustrative of the closeness between the two bends: the same separation quality, say ,could be illustrative of a poor match for two plain bends or a decent match for two complex bends. Subsequently, the estimation of the separation between two bends gives a valuable sign to the acknowledgment errand just if contrasted with some edge esteem that relies on upon bend many-sided quality. Besides, it is when all is said in done expected that not all the facial bends have the same significance in segregating between diverse subjects: given a face sweep, some of its facial bends can be fundamentally the same to some facial bends distinguished in the face outputs of distinctive people; interestingly, some facial bends can be exceptionally remarkable, in that the occasion to locate some comparative facial bends in the face outputs of distinctive people once in a while happens. Henceforth, it is important to incorporate inside of the proposed representation the idea of saliency of the facial bends, so that the higher the saliency of a bend, the 


\section{International Journal of Science and Research (IJSR) \\ ISSN (Online): 2319-7064}

Index Copernicus Value (2013): 6.14 | Impact Factor (2014): 5.611

lower the instability about the personality of the individual that is spoken to in the face filter if the bend is watched.

\section{Conclusion}

To break down the different calculation of 3D face acknowledgment through which we reason that 3D face acknowledgment explains the difficulties which found in the outcome of 2D face acknowledgment principally the enlightenment and stance issue through different methodologies. The 3D face acknowledgment methodologies are still tried on exceptionally in different approach. The information sets are expanding amid the years since better securing materials get to be accessible. On the other hand, the datasets are expanding amid the years its utilized for different methodology. The information rate is expanded it will lead to diminishing the execution of representation. So the calculations must be balanced and enhanced before they will have the capacity to handle substantial datasets with the same acknowledgment execution. The downside of most exhibited 3D face representations routines is that most calculations still regard the human face as an unbending article. This implies the systems equipped for taking care of the face representations. To contrast with $3 \mathrm{D}$ face acknowledgment idea, most 2D face .acknowledgment calculations are as of now tried on substantial datasets what's more, can deal with the extent of the information passable well. 3D face give the entire face representation like surface data that can be utilized for face acknowledgment. Another significant point of interest is that $3 \mathrm{D}$ face acknowledgment is stance invariant. In this manner, 3D face acknowledgment is still a testing yet extremely encouraging exploration range.

\section{References}

[1] A. K. Jain, M. N. Murty, and P. J. Flynn, Đata clustering: A review," ACM Comput. Surveys, vol. 31, no. 3, pp. 264-323, 1999.

[2] S. Berretti, A. Del Bimbo, and P. Pala, 3D face recognition using isogeodesic stripes," IEEE Trans. Pattern Anal. Mach. Intell., vol. 32, no. 12, pp. 21622177, Dec. 2010.

[3] A. S. Mian, M. Bennamoun, and R. Owens, An efficient multimodal 2D-3D hybrid approach to automatic face recognition," IEEE Trans. Pattern Anal. Mach. Intell., vol. 29, no. 11, pp. 1927-1943, Nov. 2007.

[4] Y. Wang, J. Liu, and X. Tang, Robust 3D face recognition by local shape difference boosting," IEEE Trans. Pattern Anal. Mach. Intell., vol. 32, no. 10, pp. 1858-1870, Oct. 2010.

[5] P. J. Phillips, P. J. Flynn, T. Scruggs, K. W. Bowyer, J. Chang, K. Hoffman, J. Marques, J. Min, and W. Worek, Đverview of the face recognition grand challenge," in Proc. IEEE Workshop on Face Recognition Grand Challenge Experiments, San Diego, CA, Jun. 2005, pp. 947-954. Olston and M .Najork , Web Crawling”, Foundations and Trends in Information Retrieval, vol. 4, No. 3 ,pp. 175-246, 2010.

[6] A. S. Mian, M. Bennamoun, and R. Owens, Keypoint detection and local feature matching for textured $3 \mathrm{D}$ face recognition," Int. J. Comput. Vis., vol. 79, no. 1, pp. 1-12, Aug. 2008.

[7] P. J. Besl and N. D. Mc Kay, A method for registration of 3-D shapes," IEEE Trans. Pattern Anal. Mach. Intell., vol. 14, no. 2, pp. 239-256, Feb. 1992.

[8] C. Xu, T. Tan, Y. Wang, and L. Quan, Combining local features for robust nose location in 3D facial data," Pattern Recognit. Lett., vol. 27, no. 13, pp. 14871494, Oct. 2006.

[9] L. G. Farkas and I. R. Munro, Anthropometric Facial Proportions in Medicine. Springfield, IL: Thomas Books, 1987.

[10] X. Lu and A. K. Jain, Multimodal Facial Feature Extraction for Automatic 3D Face Recognition, Tech. Rep. MSU-CSE-05-22, Oct. 2005.

[11] X. Lu, A. K. Jain, and D. Colbry, Matching 2.5D face scans to 3D models," IEEE Trans. Pattern Anal. Mach. Intell. vol. 28, no. 1, pp. 31-43, Jan. 2006.

[12] A. Vedaldi and B. Fulkerson, VLFeat: An Open and Portable Library of Computer Vision Algorithms 2008 [Online]. Available: http://www. vlfeat.org/

[13] M. S. Kaminer, K. A. Arndt, and J. S. Dover, Atlas of Cosmetic Surgery. Amsterdam, The Netherlands: Elsevier Health Sciences, 2008. 